\title{
Design and Analysis of a New 7-DOF Parallel Type Haptic Device : PATHOS-II
}

\author{
Keehoon Kim \\ Wan Kyun \\ Dept. of Mechanical Engineering, Pohang University of Science and Technology(POSTECH), \\ Pohang, Korea. \\ Tel : +82-54-279-2844, Fax: +82-54-279-5899, \\ E-mail : \{khk,wkchung\}@postech.ac.kr.
}

- ABSTRACT: Most tele-operation to manipulate an object consists of gripping and manipulation and two or more 6-DOF haptic devices in master side are usually used. In this article, a new simply designed 7-DOF haptic device, PATHOS II is proposed for 1-DOF gripping and 6-DOF manipulation. The merits of a parallel type haptic device such as high stiffness and accuracy are natural characteristics of PATHOS-II with optimized workspace. Due to its unique symmetric structure, the isotropic manipulability is enhanced within the reachable workspace. This parallel type haptic device can be used in applications which need high resolution, stiffness and isotropic manipulability.

KEYWORDS: Haptic Device, Parallel type, 7 DOF Robot.

\section{INTRODUCTION}

A haptic device is an instrument to convey operator's command to slave manipulator and produce sensation to make an operator feel the situation of the slave side. Recently, the number of applications of tele-operation is growing in various fields of micro manipulation, tele-surgery, virtual training, and outdoor robotics. Haptic devices can be classified into glove type[glove_type], exoskeleton type [exoskeleton_type], and pen type[pen_type] by the way of transmitting sensation or serial type[pen_type], parallel type[parallel_type], and magnetic levitation type[magnetic type] by the structure or the way of generating sensation. The type of haptic device is determined by the purpose of their required function.

In tele-operation, most of task procedure is : the operator moves the slave manipulator near the target, grasps the object, and manipulates it using two or more haptic devices. For the purpose of the task, two 6 DOF haptic devices are usually used.

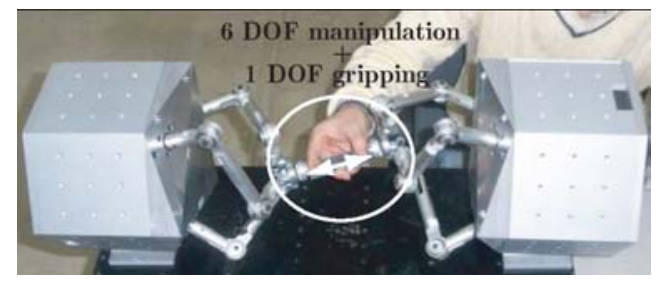

Figure 1. PATHOS-II.

However, it is redundant to use two haptic device for the task. In this article, a new parallel type haptic device for 6-DOF motion and force reflection with 1-DOF pinch grasping, PATHOS-
II(Postech pArallel Type Haptics Operating System), is proposed. Figure 1 shows that it is possible to do the task with a PATHOS-II.

The most important reason to use parallel structure in haptic device is its high stiffness compared to serial type. With this characteristic, the haptic device can not only display high force feedback but also generate elaborate motion with high accuracy. In spite of the merits, there are tradeoff like complex kinematics, small workspace, isotropy, and singularity problems.

PATHOS-II is designed simply with only revolute joints to minimize above mentioned complex kinematics. Since it has simple structure, it is easy to solve kinematics and computational load becomes lower. Especially, with only 9 sensors among 25 joints, forward kinematics of 7-DOF can be obtained as will be stated in section 2. In order to maximize the workspace of PATHOS-II, link ratio and initial orientation are optimized. The workspace covers the human haptic manipulation[khkhaptics] as will be stated in section 3. Isotropy is another important factor to determine whether the designed device can be used as a haptic device. In order to guarantee similar isotropic stiffness, PATHOS-II was designed to have symmetric structure. Active joint direction vector is also considered. As a result, uniform isotropic property of PATHOS-II is mentioned in section 4 and conclusion follows.

\section{Kinematics}

PATHOS-II was designed symmetrically with 6 legs which consist of only revolute joints and as a result, the kinematics is simple to solve. In this 
section, structure of PATHOS-II is explained and inverse and forward kinematics with 9 sensors will be shown.

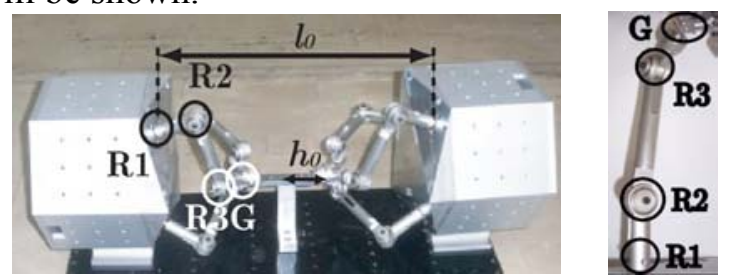

Figure 2. PATHOS-II and a leg

\subsection{System Description}

The leg of PATHOS-II has 3 revolute joints and 1 globular joint which has 3 revolute joints as shown in Figure 2.

The revolute joint at the end of a leg, $R 1$, is fixed on base plate and the globular joint is fixed on top plate. Figure 3 shows the structure of PATHOS-II. The number in circles on base plates denotes the position of leg and globular joints are also located on top plate in the same order. Then, PATHOS-II has 21 links( 3 links in each leg, 1 base plate, and 2 top plate), 25 joints( 1 globular and 3 revolute joints in a leg and a joint in top plate). From Freudenstein and Maki's method [degreesoffreedom] for spatial mechanism ${ }^{1}$

$$
\begin{aligned}
F & =6(L-J-1)+\sum_{i=1} f_{i} \\
& =6(21-25-1)+1 \times 3 \times 6+3 \times 6+1 \times 1 \\
& =7 \text { (DOF). }
\end{aligned}
$$

The first joints of each leg, R1 in Figure 2, are active joints. $h_{0}$ is half of distance between top plates in Figure 3. It is changed by an operator grasping motion. Therefore, PATHOS-II needs 6 actuators at the first joint of each leg and one between top plates for force feedback.

If $\mathrm{h}_{0}$ in Figure 3 becomes constant when an operator grips an object,

$$
\begin{aligned}
F & =6(20-24-1)+1 \times 3 \times 6+3 \times 6 \\
& =6(\mathrm{DOF}) .
\end{aligned}
$$

and 6 DOF manipulation of an object is possible.

\subsection{Inverse Kinematics}

In Figure 3, X-Y-Z coordinate at the center of base plate is the reference and $x-y-z$ coordinate fixed at the center of top plate is used to describe the pose of end effector. Those coordinates are common to every leg. One leg has the following transformation from reference coordinate to the pose of end effector.

\footnotetext{
${ }^{1} \mathrm{~F}$ is degree of freedom, $\mathrm{L}$ is number of links, $\mathrm{J}$ is number of joints, and $f_{i}$ is degree of freedom of $i$ th joint.
}

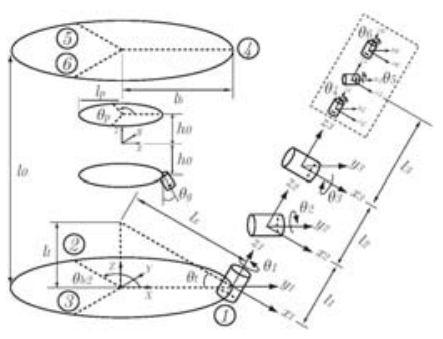

Figure 3. Structure of PATHOS-II

$$
\begin{gathered}
\mathbf{T}=\left\{T_{z}(L) R_{x}\left(\theta_{b 1}\right) R_{z}\left(\theta_{b 2}\right) T_{z}\left(l_{t}\right) R_{y}\left(\theta_{t}\right) T_{x}\left(l_{x}\right)\right\} R_{z}\left(\theta_{1}\right) \\
T_{z}\left(l_{1}\right) R_{y}\left(\theta_{2}\right) T_{z}\left(l_{2}\right) R_{x}\left(\theta_{3}\right) T_{z}\left(l_{3}\right) R_{z}\left(\theta_{4}\right) R_{y}\left(\theta_{5}\right) \\
R_{x}\left(\theta_{6}\right)\left\{R_{x}\left(\theta_{b 1}\right) T_{z}\left(-h_{0}\right) R_{z}\left(\theta_{p}\right) T_{x}\left(l_{p}\right) R_{y}\left(\theta_{g}\right)\right\}^{-1} .
\end{gathered}
$$

$\mathrm{T}$ and $\mathrm{R}$ in Eq.(1) are homogeneous transform which mean translation and rotation. $\mathrm{T}_{\mathrm{x}}$ means translation in $x$ direction and $R_{x}$ means rotation about $\mathrm{x}$ axis. $\mathrm{L}$ is 0 for 1 st to 3 rd leg and $1 \_0$ for 4th to 6th leg. $1_{0}$ is the distance between base plates. $\theta_{b 1}$ is $0^{\circ}$ for 1 st to $3 \mathrm{rd}$ leg and $180^{\circ}$ for 4th to 6th leg. Other variables in Eq.(1) are shown in Figure 3. If the system is determined, all terms of Eq.(1) are known except $\theta_{i}(i=1,2,3,4,5,6)$.

From Eq.(1), we can find the position closure and position vector, $\mathbf{a}$ in Eq.(2) is known.

$$
R_{z}\left(\theta_{1}\right) T_{z}\left(l_{1}\right) R_{y}\left(\theta_{2}\right) T_{z}\left(l_{2}\right) R_{x}\left(\theta_{3}\right) T_{z}\left(l_{3}\right)=\left[\begin{array}{ll}
x & \mathbf{a} \\
\mathbf{0} & 1
\end{array}\right] .
$$

Hereafter, orientation part and translation part of homogeneous transform will be used for $\mathrm{R}$ and $\mathrm{T}$, respectively. $\mathrm{R}$ is a $3 \times 3$ matrix and $\mathrm{T}$ is a $3 \times 1$ vector. Then, Eq.(2) becomes

$$
R_{z}\left(\theta_{1}\right)\left[T_{z}\left(l_{1}\right)+R_{y}\left(\theta_{2}\right)\left\{T_{z}\left(l_{2}\right)+R_{x}\left(\theta_{3}\right) T_{z}\left(l_{3}\right)\right\}\right]=\mathbf{a} .
$$

Eq.(3) can be represented by the following 3 equations.

$$
\begin{aligned}
R_{z}\left(\theta_{1}\right) \mathbf{C}_{1} & =\mathbf{a}, \\
\mathbf{C}_{1}-T_{z}\left(l_{1}\right) & =R_{y}\left(\theta_{3}\right) \mathbf{C}_{2}, \\
\mathbf{C}_{2}-T_{z}\left(l_{2}\right) & =R_{x}\left(\theta_{3}\right) T_{z}\left(l_{3}\right) .
\end{aligned}
$$

In matrix form, it is

$$
\begin{aligned}
& {\left[\begin{array}{ccc}
C \theta_{1} & -S \theta_{1} & 0 \\
S \theta_{1} & C \theta_{1} & 0 \\
0 & 0 & 1
\end{array}\right]\left[\begin{array}{l}
c_{1 x} \\
c_{1 y} \\
c_{1 z}
\end{array}\right]=\left[\begin{array}{l}
a_{x} \\
a_{y} \\
a_{z}
\end{array}\right],} \\
& {\left[\begin{array}{ccc}
C \theta_{2} & 0 & S \theta_{2} \\
0 & 1 & 0 \\
-S \theta_{2} & 0 & C \theta_{2}
\end{array}\right]\left[\begin{array}{l}
c_{2 x} \\
c_{2 y} \\
c_{2 z}
\end{array}\right]=\left[\begin{array}{l}
c_{1 x} \\
c_{1 y} \\
c_{1 z}
\end{array}\right],}
\end{aligned}
$$




$$
\left[\begin{array}{ccc}
1 & 0 & 0 \\
0 & C \theta_{3} & -S \theta_{3} \\
0 & S \theta_{3} & C \theta_{3}
\end{array}\right]\left[\begin{array}{l}
0 \\
0 \\
l_{3}
\end{array}\right]=\left[\begin{array}{c}
c_{2 x} \\
c_{2 y} \\
c_{2 z}-l_{2}
\end{array}\right] .
$$

From Eq.(5-6), and (7), the equivalent 6 equations are

$c_{1 z}=a_{z}$,

$\left[\begin{array}{c}C \theta_{1} \\ S \theta_{1}\end{array}\right]=\frac{1}{c_{1 x}^{2}+c_{1 y}^{2}}\left[\begin{array}{cc}c_{1 x} & c_{1 y} \\ -c_{1 y} & c_{1 x}\end{array}\right]\left[\begin{array}{l}a_{x} \\ a_{y}\end{array}\right]$,

$c_{2 y}=c_{1 y}$,

$\left[\begin{array}{c}C \theta_{2} \\ S \theta_{2}\end{array}\right]=\frac{1}{c_{2 x}^{2}+c_{2 z}^{2}}\left[\begin{array}{cc}c_{2 x} & c_{2 z} \\ c_{2 z} & -c_{2 x}\end{array}\right]\left[\begin{array}{c}c_{1 x} \\ c_{1 z}-l_{1}\end{array}\right]$,

$$
c_{2 x}=0 \text {, }
$$

$$
\left[\begin{array}{c}
C \theta_{3} \\
S \theta_{3}
\end{array}\right]=\frac{1}{l_{3}}\left[\begin{array}{c}
c_{2 z}-l_{2} \\
-c_{2 y}
\end{array}\right] .
$$

After some calculation with Eq.(8) to (13), coefficients are,

$$
\begin{aligned}
& c_{1 x}= \pm \sqrt{a_{x}^{2}+a_{y}^{2}+a_{z}^{2}}, \\
& c_{1 y}=c_{2 y}= \pm \sqrt{l_{3}^{2}-\left(c_{2 z}-l_{2}\right)^{2}} \\
& c_{1 z}=a_{z} \\
& c_{2 x}=0 \\
& c_{2 y}= \pm \sqrt{l_{3}^{2}-\left(c_{2 z}-l_{2}\right)^{2}} \\
& c_{2 z}=\left\{l_{2}^{2}-l_{3}^{2}+a_{x}^{2}+a_{y}^{2}+\left(a_{z}-l_{1}\right)^{2}\right\} / 2 l_{2} .
\end{aligned}
$$

Then, $\theta_{1}, \theta_{2}$, and $\theta_{3}$ are solved from Eq.(9), (11), and (13) with coefficients in Eq.(14).

Then, in Eq.(1), only 3 terms, $\theta_{4}, \theta_{5}$, and $\theta_{6}$ are unknown. To find them, the following equations are derived.

$$
R_{z}\left(\theta_{4}\right) R_{y}\left(\theta_{5}\right) R_{z}\left(\theta_{6}\right)=\mathbf{B}=\left[\begin{array}{cccc}
b_{11} & b_{12} & b_{13} & 0 \\
b_{21} & b_{22} & b_{23} & 0 \\
b_{31} & b_{32} & b_{33} & 0 \\
0 & 0 & 0 & 1
\end{array}\right] \text {. }
$$

The matrix $\mathrm{B}$ is known from Eq.(1). After multiplying $R_{z}\left(\theta_{4}\right)^{-1}$,

$$
\begin{aligned}
\theta_{4} & =\tan ^{-1}\left(\frac{b_{23}}{b_{13}}\right), \\
{\left[\begin{array}{c}
C \theta_{5} \\
S \theta_{5}
\end{array}\right] } & =\left[\begin{array}{c}
b_{33} \\
b_{13} C \theta_{4}+b_{23} S \theta_{4}
\end{array}\right], \\
{\left[\begin{array}{c}
C \theta_{6} \\
S \theta_{6}
\end{array}\right] } & =\left[\begin{array}{l}
-b_{12} S \theta_{4}+b_{22} C \theta_{4} \\
-b_{11} S \theta_{4}+b_{21} C \theta_{4}
\end{array}\right] .
\end{aligned}
$$

These give $\theta_{4}, \theta_{5}$, and $\theta_{6}$ and this procedure is applied to every leg.

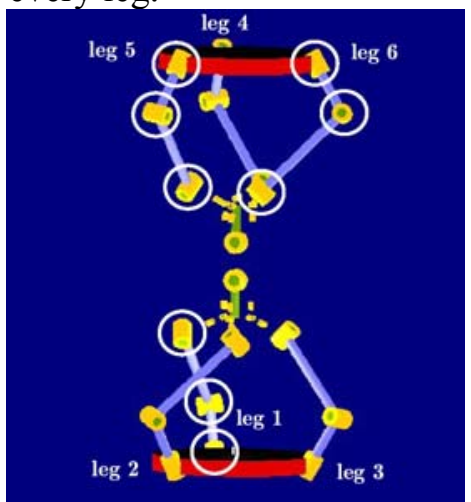

Figure 4. The location of sensors

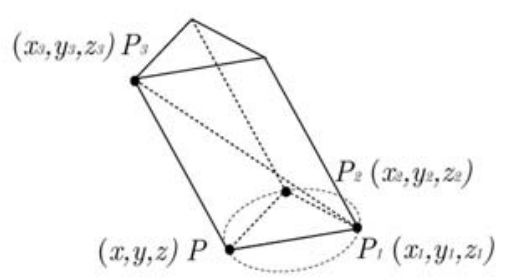

Figure 5. Top plate and positions of globular joints of $1 \mathrm{st}\left(P_{3}\right), 5 \operatorname{th}\left(P_{2}\right), 6 \operatorname{th}\left(P_{1}\right)$, and 4th leg(P)

\subsection{Forward Kinematics}

Forward kinematics is to find the pose of top plate and top plate length, $h_{0}$ in Eq.(1) from the sensor information. PATHOS-II uses 9 sensors to solve 7-DOF forward kinematics.

The locations of sensors are shown in Figure 4. In Eq.(1), $\mathbf{T}$ and $T_{z}\left(-h_{0}\right)$ on the right hand side for 1st, 5th, and 6th leg are unknown. The positions of globular joints of 1st, 5th, and 6th leg, a in Eq.(2), which has 3 sensors can be calculated.

Then, Figure 5 shows the top plate and vertices mean the positions of globular joints of each leg. In other words, $P_{1}\left(x_{1}, y_{1}, z_{1}\right), P_{2}\left(x_{2}, y_{2}, z_{2}\right), P_{3}\left(x_{3}, y_{3}, z_{3}\right)$, and $P(x, y, z)$ are positions of globular joints of 5th, 6 th, and 1st leg, respectively. First, from the points, $P_{1}, P_{2}$, and $P_{3}, h$ can be calculated as followings.

$$
\begin{aligned}
h & =\overline{P P_{3}} \\
& =\sqrt{\left(x_{1}-x_{3}\right)^{2}+\left(y_{1}-y_{3}\right)^{2}+\left(z_{1}-z_{3}\right)^{2}-3 r^{2}} .
\end{aligned}
$$

The point $\mathrm{P}$ can be calculated as 


$$
\begin{aligned}
& {\left[\begin{array}{lll}
2\left(x_{2}-x_{1}\right) & 2\left(y_{2}-y_{1}\right) & 2\left(z_{2}-z_{1}\right) \\
2\left(x_{3}-x_{2}\right) & 2\left(y_{3}-y_{2}\right) & 2\left(z_{3}-z_{2}\right) \\
2\left(x_{1}-x_{3}\right) & 2\left(y_{1}-y_{3}\right) & 2\left(z_{1}-z_{3}\right)
\end{array}\right]\left[\begin{array}{l}
x \\
y \\
z
\end{array}\right] } \\
= & {\left[\begin{array}{c}
x_{2}^{2}+y_{2}^{2}+z_{2}^{2}-\left(x_{1}^{2}+y_{1}^{2}+z_{1}^{2}\right) \\
3 r^{2}-h^{2}+\left(x_{3}^{2}+y_{3}^{2}+z_{3}^{2}\right)-\left(x_{2}^{2}+y_{2}^{2}+z_{2}^{2}\right) \\
h^{2}-3 r^{2}+\left(x_{1}^{2}+y_{1}^{2}+z_{1}^{2}\right)-\left(x_{3}^{2}+y_{3}^{2}+z_{3}^{2}\right)
\end{array}\right] . }
\end{aligned}
$$

Since $P, P_{1}$, and $P_{2}$, are points on a plane, the center of the points and the orientation of the plane can be derived. Finally, the pose of the top plate can be found.

\section{Workspace and Optimization}

After the kinematic solution, we need to consider workspace. In fact, though a number of parallel type haptic devices have been designed with their own optimization technique, parallel type has so many design factors and complex characteristics that there is no general cost function, fundamentally for the optimization of workspace.

\subsection{Link Ratio and Initial Orientation Optimization}

Optimization of PATHOS-II is to maximize workspace under constraint of link lengths. The most important factor which affects the volume of workspace is link ratio and initial orientation. Therefore, in order to maximize the workspace, cost function which is dependent on $l_{2}, l_{3}, l_{p}$, and initial orientation about $\mathrm{z}$ axis is selected as the following.

$$
G\left(l_{2}, l_{3}, l_{p}, R_{z}\right)=\frac{\Delta L_{x}+\Delta L_{y}+\Delta L_{z}}{\left(l_{2}+l_{3}+l_{p}\right) / 3} .
$$

First, system parameters except $l_{2}, l_{3}, l_{p}$, and $R_{z}$ are fixed as shown in Table 1. $\Delta L_{x}, \Delta L_{y}$, and $\Delta L_{z}$ denote movable ranges in $\mathrm{x}, \mathrm{y}$, and $\mathrm{z}$ direction in which inverse kinematics solution exists continuously. The numerator means the workspace and the denominator keeps the cost function from being larger as links become longer.

Table 1. Given pose to find $\Delta L_{x}, \Delta L_{y}$, and $\Delta L_{z}$

\begin{tabular}{|c|c|c|c|}
\hline$l_{0}$ & 50.0 & $x$ & 0.0 \\
\hline$l_{b}$ & 10.0 & $y$ & 0.0 \\
\hline$l_{1}$ & 7.0 & $z$ & 25.0 \\
\hline$h_{0}$ & 7.0 & $R_{x}$ & 0.0 \\
\hline$\Delta \theta_{b 1}$ & $180^{\circ}$ & $R_{y}$ & 0.0 \\
\hline
\end{tabular}

\begin{tabular}{|c|c|c|c|}
\hline$\Delta \theta_{b 2}$ & $120^{\circ}$ & $\theta_{g}$ & $0^{\circ}$ \\
\hline$\Delta \theta_{p}$ & $120^{\circ}$ & $\theta_{t}$ & $30.0^{\circ}$ \\
\hline$l_{x}$ & $l_{b} / \cos \theta_{t}$ & $l_{t}$ & $l_{b} / \sin \theta_{t}$ \\
\hline
\end{tabular}

The value of cost function is calculated by increasing $l_{2}, l_{3}, l_{p}$, and $\mathrm{R}_{z}$ with the steps within ranges in table 2. Then, if collision between legs or exceeding the joint angle limit is happened during finding $\Delta L_{x}, \Delta L_{y}$, and $\Delta L_{z}$ in Eq.(21), the results are excluded. Cost function was maximized when $l_{2}=14.0, l_{3}=6.0$, $l_{4}=2.0$, and $R_{z}=30.0^{\circ}$.

Table 2. Given ranges of variables to find $\Delta L_{x}, \Delta L_{y}$, and $\Delta L_{z}$

\begin{tabular}{|c|c|c|c|}
\hline Variables & Lowest limit & Upper limit & Step \\
\hline$l_{2}$ & 0.0 & 20.0 & 1.0 \\
\hline$l_{3}$ & 0.0 & 20.0 & 1.0 \\
\hline$l_{p}$ & 0.0 & 20.0 & 1.0 \\
\hline$R_{z}$ & $0^{\circ}$ & $120^{\circ}$ & $15^{\circ}$ \\
\hline
\end{tabular}

\subsection{Workspace}

Now, link ratio and initial pose are determined after the optimization of cost function of Eq.(21). However, PATHOS-II is designed to change the length $l_{0}$ in Figure 3 . Figure 6 shows the workspace when $l_{0}$ is changed from 40.0 to 55.0. The workspace increases when $l_{0}$ becomes smaller.

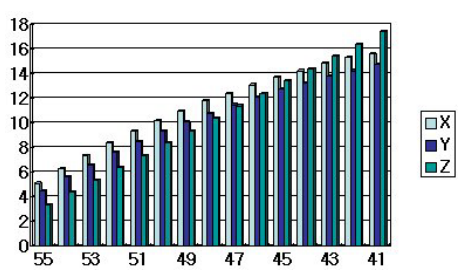

(a)

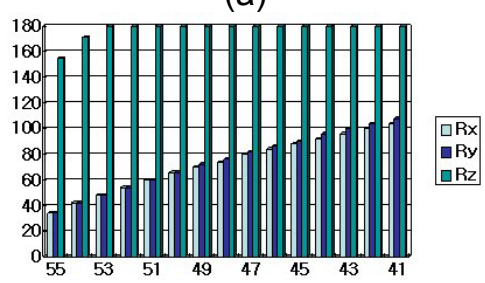

(b)

Figure 6. (a) : Workspace for fixed initial orientation, (b) : Workspace for fixed position

$\Delta L$ and $\Delta \theta$ in Figure 6 denote the workspace in which inverse kinematics is obtained continuously. 
For example, $\Delta L$ in Figure 6.(a) is the movable range of each direction when orientation is fixed in initial pose. However, for small $l_{0}$, space for human grip decreases and collision between human hand and legs is expected. $l_{0}=45.0$ is suitable to avoid collision and maximize workspace.

In fact, the workspace can be increased as the links become longer. So, the workspace is compared to the haptic device size. The dimension of PATHOS-II is a cylinder shape of $20.0 \mathrm{~cm}$ radius and $45.0 \mathrm{~cm}$ height and the workspace is about a sphere of $14.0 \mathrm{~cm}$ diameter which can cover the human haptic manipulation range[khkhaptics]. However, this reachable workspace can not be used because of manipulability and isotropy. In the case of PATHOS-II, this reachable workspace can be almost used in the view point of isotropy. It will be explained in the next section.

\section{Isotropy}

Another important requirement for a good haptic device is isotropy which makes handle move to any direction an operator wants or guarantees similar stiffness of an haptic device toward every direction.

$$
\dot{\mathbf{x}}=\mathbf{J} \dot{\mathbf{q}}
$$

where

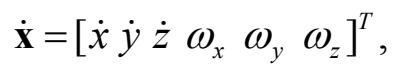

$$
\begin{aligned}
& \mathbf{J}=\left[\begin{array}{ll}
\mathbf{J}_{p}^{T} & \mathbf{J}_{o}^{T}
\end{array}\right]^{T}, \\
& \dot{\mathbf{q}}=\left[\begin{array}{llllll}
\dot{q}_{1} & \dot{q}_{2} & \dot{q}_{3} & \dot{q}_{4} & \dot{q}_{5} & \dot{q}_{6}
\end{array}\right]^{T} \text {. } \\
& \delta \mathbf{q} \cdot \tau=\delta \mathbf{x}^{T} \cdot \mathbf{F}, \\
& \tau=\mathbf{J}^{T} \cdot \mathbf{F} .
\end{aligned}
$$

Eq.(22) and (23) show velocity and force relations between task space and joint space. $\dot{\mathbf{x}}$ and $\dot{\mathbf{q}}$ mean velocities of task coordinate and active joint angles. Though PATHOS-II has 7 actuators, since $h_{0}$ is constant when an operator grips an object, it is reasonable to consider 6-DOF spatial task space. The jacobian, $\mathbf{J}$, is decomposed to $\mathbf{J}_{p}$ and $\mathbf{J}_{o}$ which are $3 \times 6$ matrices related to velocity and angular velocity since position and orientation have different dimensions. After singular value decomposition, condition number which is the ratio of maximum and minimum singular values means isotropy of velocity and angular velocity or force and moment. For example, if condition number isclose to 1 , system is isotropic at the attitude. If its condition number becomes large or singular, the haptic device is not a structure any more, since force reflecting is not available. To be a nice haptic device, it should be isotropic in every attitude in its workspace.

Though PATHOS-II is designed to have symmetric structure for isotropy, the stiffness in $\mathrm{Z}$ direction in Figure 3 can be lower than other directions if the direction vector of active joints is aligned to $\mathrm{z}$ direction. So, $\theta_{t}$ needs to be determined to make PATHOS-II have similar stiffness in every direction and $\theta_{t}$ is selected as $30^{\circ}$.

Condition numbers of PATHOS-II in 6 plane are shown in Figure 7 and 8 which are obtained from $\mathbf{J}_{p}$ and $\mathbf{J}_{o}$, respectively. (b), (d), and (f) are projection images of (a), (c), and (e) to the plane. For example, to draw (a) and (b) in Figure 7, condition number of $\mathbf{J}_{p}$ is calculated from the initial pose of PATHOS-II in Table 3 to every point by increasing $\mathrm{x}$ and $\mathrm{y}$ by 0.1 in reachable workspace.

From Figure 7 and 8, the condition number is less than 5 for the most part of workspace. However, the condition numbers increase sharply near workspace boundaries. In $x-y$ plane, reasonable workspace as a haptic device is a circle shape of diameter $12 \mathrm{~cm}$. In $y-z$ plane, PATHOS-II is isotropic in reachable workspace of which the length of the major and minor axis are $14 \mathrm{~cm}$ and $12 \mathrm{~cm}$, respectively. In $\mathrm{x}-\mathrm{z}$ plane, the workspace in which $\mathrm{x}$ is larger than $6 \mathrm{~cm}$ is not available, therefore, the workspace is almost the same as the one in $\mathrm{y}-\mathrm{z}$ axis.

Table 3 : Initial pose of PATHOS-II

\begin{tabular}{|c|c|c|c|c|c|c|}
\hline$x$ & $y$ & $z$ & $R_{x}$ & $R_{y}$ & $R_{z}$ & $l_{0}$ \\
\hline 0.0 & 0.0 & 22.5 & $0.0^{\circ}$ & $0.0^{\circ}$ & $30.0^{\circ}$ & 45.0 \\
\hline
\end{tabular}

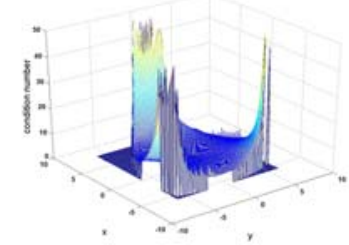

(a)

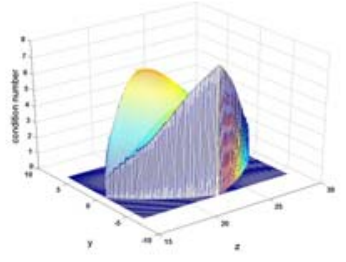

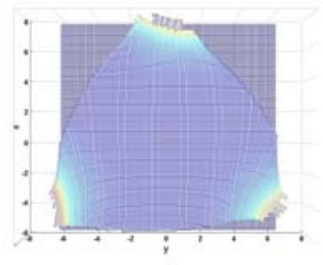

(b)

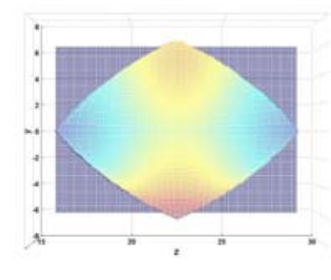


(c)

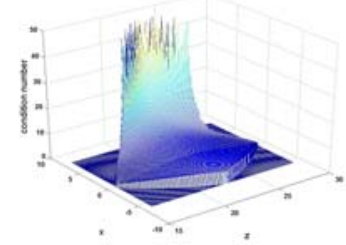

(e) (d)

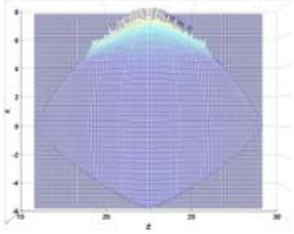

(f)
Figure 7. Condition numbers of $\mathbf{J}_{p}$ (a) : $\mathbf{x}-\mathbf{y}$, (b) : projection (a) to $x-y$ plane ,(c) : y-z,(d) : projection (c) to $y-z$ plane, ,(e) : $x-z$,(f): projection (e) to $x-z$ plane

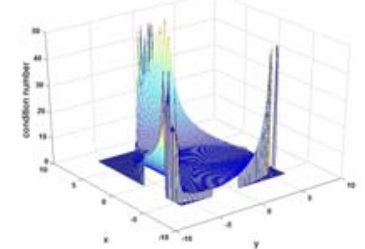

(a)

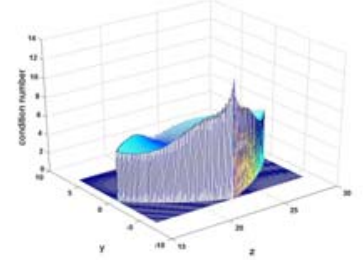

(c)

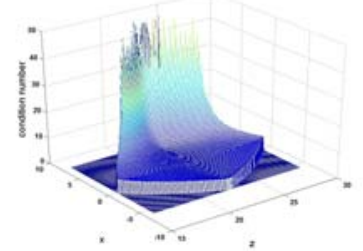

(e)

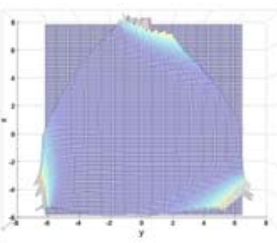

(b)

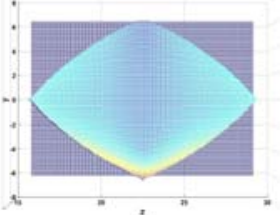

(d)

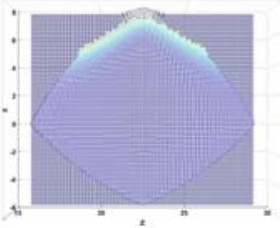

(f)
Figure 8. Condition numbers of $\mathbf{J}_{o}$ (a) : $x-y$, (b) : projection (a) to $x-y$ plane ,(c) : y-z,(d) : projection (c) to $y-z$ plane, (e) : $x-z$,(f): projection (e) to $x-z$ plane

\section{CONCLUSIONS}

The proposed haptic device PATHOS-II enables 6-DOF manipulation and 1-DOF gripping and its inverse kinematics was solved using 9 sensors.

The workspace was optimized to maximize it under the constraint of link length. The link ratio and initial orientation was used to do this.

Another strong point of PATHOS-II is the enhanced isotropic manipulability in most reachable workspace with simple kinematics. This was possible because of its symmetric structure.
The condition number was shown to have similar stiffness in reachable workspace.

It is expected that PATHOS-II can be used effectively as a haptic device for manipulating an object in many applications, including outdoor robotics.

\section{Acknowledgement}

This work was supported by grant No. 2000-230200-008-3 from the Basic Research Program of the Korea Science \& Engineering Foundation and No. 02-PJ3-PG6-EV04-0003 from of the Korea Health 21 R\&D Project, Ministry of Health \& Welfare, Republic of Korea.

\section{REFERENCES}

[glove type] M. Bouzit, G. Burdea, G. Popescu, and R. Boian, "The Rutgers MasterII-New Design ForceFeedback Glove, "IEEE/ASME Trans. on Mechatronics, vol. 7, pp. 256-263, June, 2002.

[exoskeleton_type] Sooyong Lee, Jangwook Lee, Woojin Chung, Munsang Kim, Chongwon Lee, and Mignon Park," A new exoskeleton-type masterarm with force reflection : Controller and integration," Proc. of 1999 IEEE/RSJ International Conference on Intelligent Robots and Systems, pp. 1438--1443, Victoria, Canada, October, 1999.

[pen_type] T. Massie, K. Salisbury, "PHANToM Haptic Interface: A Device for Probing Virtual Objects," ASME Journal of Dynamic System and Control, New York, pp.295-299, 1994.

[parallel_type] J.H. Lee, K.S. Eom, B.J. Yi, and I.H. Suh, "Design of A New 6-DOF Parallel Haptic Device," Proc. Of the IEEE International Conference on Robotics and Automation, pp.886-891, Seoul, Korea, May 21-26, 2001.

[magnetic_type] P. Berkelman and R. Hollis, "Lorentz magnetic levitation for haptic interaction: Device design, performance, and integration with physical simulations," The International Journal of Robotics Research, vol. 19, pp. 644-667, July, 2000.

[degreesoffreedom] F. Freudenstein, E.R. Maki, “ The creation of mechanisms according to kinematic structure and function," Environment and Planning B, 1979, pp. 375-391, Vol 6.

[khkhaptics] Keehoon Kim, Y. Youm, and W.K. Chung, "Human Kinematic Factor for Haptic Manipulation : The Wrist to Thumb," 10th International Symposium on Haptic Interfaces for Virtual Environment and Teleoperator System, March 24-25, 2002, pp. 319-326.

[khkmaster] Keehoon Kim, "Design and Control of 6DOF Parallel Manipulator for Haptic Display," Thesis of Master Degree, Pohang University of Science and Technology 2001. 